\title{
The psychology and neuroscience of depression and anxiety: towards an integrative model of emotion disorders
}

\author{
Andrew H. Kemp and Kim L. Felmingham \\ University of Sydney, Australia
}

\begin{abstract}
Current theoretical models of emotion highlight the importance of distinguishing depression and anxiety. The present article critically evaluates a number of these models and provides a practical framework that could be applied in future studies to better understand the neural substrates that contribute to variation in anxiety and depressed mood. One influential model, the tripartite model, suggests that depression and anxiety can be distinguished on the basis of anhedonia and hyperarousal. Yet this model is based predominantly on questionnaire data. A more direct and powerful method to test this model is to identify biological markers of arousal and anhedonia. Other influential models, such as the approach-withdrawal and valence-arousal models, are based on biological measures and integrate the concept of arousal - but have generally restricted empirical enquiry into resting state paradigms, without an integrative approach to explore concurrent physiological arousal using autonomic measures, or to extend into emotion processing paradigms. The authors propose a practical framework that will have significant implications for theoretical models of depression and anxiety including integration of influential models of emotion and advancement of the knowledge base, clarification of the neurobiological specificity of depression and anxiety and identification of overlapping and distinctive features of these disorders. Keywords: depression, anxiety, models of emotion, specificity, distinctive features.
\end{abstract}

Received 9 October 2008; received in revised form 31 October 2008; accepted 3 November 2008. Available online 22 December 2008

\section{Introduction}

How people process positive and negative stimuli is central to theories of emotion, and may be the key component in vulnerability factors governing risk for depression and anxiety (Davidson, Pizzagalli, Nitschke, \& Kalin, 2003). Depression and anxiety are commonly experienced in the general population and may significantly impair psychosocial function. In their extreme form these negative affective states develop into clinical depression and anxiety - the most commonly experienced psychiatric disorders today. While these disorders are often characterised as distinct phenomena, they co-occur in up to half the cases with either disorder (Sartorius, Ustun, Lecrubier, \& Wittchen, 1996). Given the high comorbidity between these disorders, the relation between them has received much attention and a number of theoretical models dealing with this relationship have been proposed (Davidson, 1992; Wendy Heller, 1993; W. Heller, Nitschke, Etienne, \& Miller, 1997; Watson

Andrew H. Kemp, and Kim L. Felmingham, Brain Dynamics Centre, University of Sydney and Westmead Hospital, Westmead, NSW Australia 2145, Phone: +612 9845 8195, Fax: +61 29845 8190. E-mail: akemp@usyd. edu.au. Correspondence regarding this article should be directed to A. H. Kemp at this same adress. et al., 1995a, 1995b) (see Shankman \& Klein, 2003 for a review). The aim of the present article is to critically evaluate a number of these models and provide a practical framework that could be applied in future studies to better understand the neural substrates that contribute to variation in, and comorbidity between anxiety and depressed mood.

\section{Theoretical Models}

Key theoretical models are discussed below and a summary of these models and their limitations is provided in Table 1.

\section{The tripartite model}

In the tripartite model, overlapping and distinctive behavioural features of these disorders are highlighted, such that depression and anxiety are linked through a nonspecific distress factor, and distinguished by anhedonia (specific to depression) and heightened arousal (anxiety) (Watson, Clark et al., 1995; Watson, Weber et al., 1995). The tripartite model highlights explicitly that while less severe depression and anxiety may not be able to be distinguished, more severe forms will be distinguished on the basis of anhedonia and arousal. However, a significant weakness of this model is its reliance on self-report questionnaire data, highlighting the need for more objective measures to test and validate this theoretical model. Reliance on such 
Table 1. Summary of key models.

\begin{tabular}{lll}
\hline Model & Key Concepts & Limitations \\
\hline Tripartite model $^{1}$ & $\begin{array}{l}\text { Depression (anhedonia) \& Anxiety (arous- } \\
\text { al) specific factors } \\
\text { data }\end{array}$ & \\
& $\begin{array}{l}\text { Depression }(\downarrow \text { approach, } \downarrow \text { left frontal } \\
\left.\text { EEG activity }{ }^{4}\right) \& \text { Anxiety }(\uparrow \text { withdrawal, } \\
\uparrow \text { right frotal EEG activity) }\end{array}$ & $\begin{array}{l}\text { Supporting evidence largely restricted to } \\
\text { resting state EEG data }\end{array}$ \\
& $\begin{array}{l}\text { Arousal factor will differentiate depres- } \\
\text { sion \& anxiety (Depression: } \downarrow \text { right } \\
\text { parieto-temporal region, Anxiety: } \uparrow)\end{array}$ & As above \\
\hline Valence-arousal model ${ }^{3}$ & & \\
\hline
\end{tabular}

Notes: ${ }^{1}$ See Watson et al., 1995a, 1995b; ${ }^{2}$ See Davidson, 1992; Mathersul et al., 2008; ${ }^{3}$ See Heller, 1993; Heller, Nitschke, Etienne, \& Miller, 1997; Mathersul et al., 2008; ${ }^{4}$ EEG: electroencephralogram.

data severely restricts the inferences that can be made about the processes and mechanisms underlying emotion (Davidson, Pizzagalli, Nitschke, \& Kalin, 2003). Indeed, Kemp and colleagues have demonstrated that while electrophysiological and autonomic activity are sensitive enough to measure the impact of a single dose of a commonly prescribed antidepressant on viewing of emotional images, no differences were observed on behavioural ratings of these images (Kemp, Gray, Silberstein, Armstrong, \& Nathan, 2004).

\section{Neurobiological models}

The proposal that anhedonia and hyperarousal may help in distinguishing anxiety and depression is supported by convergent neurobiological-based models: the approach-withdrawal and valence-arousal models of emotion (Davidson, 1992; Wendy Heller, 1993; W. Heller, Nitschke, Etienne, \& Miller, 1997). The approach system controls appetitive and other goal-directed behaviour, while the withdrawal system facilitates behaviour that removes an organism from sources of aversive stimulation. While depression has been characterised by reduced approach, anxiety has been characterised by increased withdrawal activity. The valence-arousal model postulates that the degree of arousal will further differentiate depression and anxiety. While all three models characterize depression as a deficit in positive affect, the tripartite model and the valence-arousal model highlight arousal as an additional distinguishing factor between depression and anxiety.

The neurobiological systems include left and right prefrontal cortex (implicated in approach and withdrawal, respectively) and the right parieto-temporal region (arousal). Interestingly, current and previously depressed patients have been reported to display lower left frontal electroencephalogram (EEG) activation than healthy participants without depression (ie. reduced approach), while individuals with anxiety display higher right frontal EEG activity (ie. enhanced withdrawal) (Davidson, Marshall, Tomarken, \& Henriques, 2000; Debener et al., 2000; Diego, Field, \& Hernandez-Reif, 2001; Mathersul, Williams, Hopkinson, \& Kemp, 2008; but see also Papousek \& Schulter, 2002; Reid, Duke, \& Allen, 1998). The valence-arousal model postulates that depression and anxiety will display decreased and increased activity in the right parieto-temporal region (Wendy Heller, 1993; W. Heller, Nitschke, Etienne, \& Miller, 1997), respectively. The role of the right parieto-temporal region in arousal processes is supported by studies on brain damaged patients, dichotic listening studies and the noradrenaline system (Oke, Keller, Mefford, \& R., 1978; Robinson, 1979) (Heller, 1993; Oke et al., 1978; Robinson, 1979). However, the approach-withdrawal and valence-arousal models are also problematic in that contradictory data has been reported, possibly due to employment of primarily resting state EEG data (as highlighted in Allen \& Kline, 2004). It is possible that collection of resting state data is insufficient to challenge the impaired circuitry in depression and anxiety and that paradigms which tap into the processing of emotion stimuli will provide more useful information relating to distinguishing between depression and anxiety (i.e. impaired emotional circuitry in participants with depression and anxiety may only appear when the circuitry is challenged with an appropriate task). In addition, restricting biological measures to collection of EEG data limits ones focus to dynamic changes in scalp brain electrical activity. Critically, meta-analysis of 65 PET and $\mathrm{fMRI}$ studies (Wager, Phan, Liberzon, \& Taylor, 2003) has provided only limited support for the proposal that emotion is lateralised according to these models. However, it should be noted that this meta-analysis only focused on emotional processing in healthy controls rather than patients with depression and anxiety. 


\section{Critical Issues}

A number of critical issues in the emotion field relevant to distinguishing depression and anxiety are responsivity to positive versus negative stimuli, automaticity versus controlled processing, temporal versus spatial dimensions of neural processes and identification of objective markers of risk in less severe samples. With respects to responsivity to positive and negative stimuli, depressed patients display a deficit in recognizing positive emotions and tend to give lower ratings for positive emotions (e.g. Surguladze et al., 2005), consistent with the tripartite model that depressed patients are distinguished by anhedonia. By contrast, individuals with anxiety may display heightened responsivity to negative stimuli (or a negativity bias) (Kaviani et al., 2004; Larson, Nitschke, \& Davidson, 2007), highlighting the need for positive and negative stimuli in studies of emotion perception and experience. Regarding automaticity versus controlled processing, anxiety may display a non-conscious attentional bias, while depression may display an attentional bias under conditions that allow elaborative processing (Mogg \& Bradley, 2005), although the majority of studies that lead to this suggestion are based on behavioural data (but see Williams et al., 2007).

In addition to prefrontal and parieto-temporal cortical regions, other more specific brain regions of interest (able to be identified using neuroimaging techniques such as fMRI) include the anterior cingulate cortex (attentional and emotional processing, as part of the medial PFC), ventromedial prefrontal cortex (negative affect), amygdala (approach and withdrawal motivational tendencies), ventral striatum - including the caudate, putamen and the nucleus accumbens (involved in positive affect) -, the hippocampus (the context-regulation of affect), and the insula (visceral representation of stimuli) (Davidson \& Irwin, 1999; Davidson, Pizzagalli, Nitschke, \& Putnam, 2002; Phan, Wager, Taylor, \& Liberzon, 2002; Zald, Mattson, $\&$ Pardo, 2002). Specific questions relating to these underlying structures remain to be clarified. For example, it is unclear whether deep lying structures such as the amygdala are specific to anxiety (i.e. is amygdala activation specific to the emotion of fear (Phan, Wager, Taylor, \& Liberzon, 2002) or arousal processing (Bechara et al., 1995; Williams et al., 2001), or whether activation is observed in depression as well as anxiety (i.e. is amygdala activation a reaction to salient stimuli (Davis \& Whalen, 2001).

Researchers have speculated that amygdala activation is observed in depression because of the frequent comorbidity with anxiety (Davidson, Pizzagalli, Nitschke, \& Putnam, 2002), while others have speculated that such activation may underlie the ruminative tendencies observed in a depressed patient (Drevets, 2001). Preliminary evidence from our laboratory found evidence for reduced medial prefrontal activation and suppression of amygdala activation in patients with post traumatic stress disorder (PTSD) with comorbid depression relative to PTSD patients without depression, suggesting that heightened amygdala activation may be associated with anxiety rather than depression per se (Kemp et al., 2007). Clarification of these responses by inclusion of depressed patients without anxiety, as well as non-clinically depressed and anxious participants as additional controls remains to be conducted. Finally, while more objective measures may help distinguish the specificity of depression and anxiety in less severe disorders, an outstanding question is whether biological markers of anhedonia and arousal can be detected in nonclinical participants and whether these are the same as those identified in more severe forms of these disorders, as is suggested by the proposal that the clinical disorders are a manifestation of a syndrome discernable in healthy participants (Judd, Schettler, \& Akiskal, 2002; Lovibond \& Lovibond, 1995; but see also Parker, 2000).

\section{Towards a better understanding of emotion}

\section{Integration of convergent theoretical models}

Converging models of depression and anxiety highlight the overlapping and distinctive features associated with these conditions, yet they remain separate and have not been adequately integrated (Davidson, 1992; Wendy Heller \& Nitscke, 1998; Watson et al., 1995a, 1995b; Williams, 2006). More specifically, the tripartite model is primarily based on questionnaire content and self-report information (Watson et al., 1995a, 1995b) and this approach has been criticised for failing to reveal the biological processes and mechanisms underlying emotion (Davidson, Pizzagalli, Nitschke, \& Kalin, 2003); the approach-withdrawal and valencearousal models are primarily based largely on EEG data and resting state activity (Allen \& Kline, 2004), and recent meta-analyses suggest that lateralisation of emotional activity is more complex and region-specific than predicted by these models (Wager, Phan, Liberzon, $\&$ Taylor, 2003); evidence further suggests that major depressive disorder (MDD) and generalised anxiety disorder (GAD) can be distinguished on the basis of nonconscious and conscious emotion processing (Mogg \& Bradley, 2005; Williams et al., 2007).

\section{Integration of data}

Data integration is well placed to achieve some of the core goals of neuropsychiatric research, including quantification of individual differences, comparison of performance to matched controls and the provision of a robust framework for clinical assessment (Gordon, Cooper, Rennie, Hermens, \& Williams, 2005). This approach will enable direct testing and extension of prevailing theoretical models in comprehensive ways 
not previously achieved. For example, our laboratory has highlighted the utility of simultaneous recording of neuroimaging data and autonomic data (Williams et al., 2001), reporting that distinct neural substrates may underpin the experiential versus factual processing of fear in the human brain, such that amygdala-medial frontal activity is only observed in the presence of skin conductance responses (SCRs), while hippocampuslateral frontal activity is only observed in the absence of SCRs. This approach will be crucial for distinguishing the specificity of amygdala activation during emotion processing in depression and anxiety.

\section{Clarification of biological bases}

Future classification systems such as the Diagnostic and Statistical Manual of Mental Disorders, must place greater emphasis on the biological mechanisms of disorders such as depression and anxiety (Kupfer, First, \& Regier, 2002). Research should consider focusing on the biological bases for major depressive disorder (MDD) and generalised anxiety disorder (GAD) in particular, considering that (1) MDD is the most common type of clinical depression, (2) GAD is the anxiety disorder most often co-morbid with MDD, (3) MDD and GAD share the vulnerability trait of negative emotionality (see Moffitt et al., 2007 for discussion), as highlighted in the tripartite model. In addressing the biological specificity (or otherwise) of depression (and anxiety), clarification of these issues may contribute to the development of an etiologically based, scientifically sound classification.

\section{Conclusion}

In conclusion, we suggest that key models relating to the specificity of anxiety and depression should be tested using a comprehensive range of biological measures, to examine responses to positively and negatively valenced stimuli, non-conscious (automaticity) versus conscious (controlled) emotion processing, temporal versus spatial dimensions of neural processes and different measures of arousal. Such research would help to determine a differential brain-behavior basis of anxiety and depression and help to identify objective markers of risk for these conditions and their early development.

\section{Acknowledgements}

This research was supported by an NHMRC Project Grant (464863) and an ARC Discovery Project (DP0987332). AHK is supported by a National Health and Medical Research Council (NHMRC) Peter Doherty Fellowship (358770) and KF by a NHMRC Australian Clinical Research Fellowship (358676). The authors would like to thank Professors Lea Williams and Richard Bryant for their guidance and intellectual enthusiasm.

\section{References}

Allen, J. J., \& Kline, J. P. (2004). Frontal EEG asymmetry, emotion, and psychopathology: the first, and the next 25 years. Biological Psychology, 67(1-2), 1-5.

Bechara, A., Tranel, D., Damasio, H., Adolphs, R., Rockland, C., \& Damasio, A. R. (1995). Double dissociation of conditioning and declarative knowledge relative to the amygdala and hippocampus in humans. Science, 269, 1115-1118.

Davidson, R. J. (1992). Anterior cerebral asymmetry and the nature of emotion. Brain Cogn, 20(1), 125-151.

Davidson, R. J., \& Irwin, W. (1999). The functional neuroanatomy of emotion and affective style. Trends in Cognitive Sciences, 3(1), 11-21.

Davidson, R. J., Marshall, J. R., Tomarken, A. J., \& Henriques, J. B. (2000). While a phobic waits: regional brain electrical and autonomic activity in social phobics during anticipation of public speaking. Biological Psychiatry, 47(2), 85-95.

Davidson, R. J., Pizzagalli, D., Nitschke, J. B., \& Kalin, N. H. (2003). Parsing the subcomponents of emotion and disorders of emotion: Perspectives from affective neuroscience. In R. J. Davidson, K. R. Scherer \& H. H. Goldsmith (Eds.), Handbook of Affective Sciences (pp. 8 - 24). New York: Oxford University Press.

Davidson, R. J., Pizzagalli, D., Nitschke, J. B., \& Putnam, K. (2002). Depression: perspectives from affective neuroscience. Annual Review of Psychology, 53, 545-574.

Davis, M., \& Whalen, P. J. (2001). The amygdala: vigilance and emotion. Molecular Psychiatry, 6(1), 13-34.

Debener, S., Beauducel, A., Nessler, D., Brocke, B., Heilemann, H., \& Kayser, J. (2000). Is resting anterior EEG alpha asymmetry a trait marker for depression? Findings for healthy adults and clinically depressed patients. Neuropsychobiology, 41(1), 31-37.

Diego, M. A., Field, T., \& Hernandez-Reif, M. (2001). CES-D depression scores are correlated with frontal EEG alpha asymmetry. Depression and Anxiety, 13(1), 32-37.

Drevets, W. C. (2001). Neuroimaging and neuropathological studies of depression: implications for the cognitive-emotional features of mood disorders. Current Opinion in Neurobiology, 11(2), 240-249.

Gordon, E., Cooper, N., Rennie, C., Hermens, D., \& Williams, L. M. (2005). Integrative neuroscience: the role of a standardized database. Clinical EEG \& Neuroscience, 36(2), 64-75.

Heller, W. (1993). Neuropsychological mechanisms of individual differences in emotion, personality, and arousal. Neuropsychology, 7, 476-489

Heller, W., Nitschke, J. B., Etienne, M. A., \& Miller, G. A. (1997). Patterns of regional brain activity differentiate types of anxiety. Journal of Abnormal Psychology, 106(3), 376-385.

Heller, W., \& Nitscke, J. B. (1998). The puzzle of regional brain activity in depression and anxiety: The importance of subtypes and comorbidity. Cognition and Emotion, 12(3), 421-447.

Judd, L. L., Schettler, P. J., \& Akiskal, H. S. (2002). The prevalence, clinical relevance, and public health significance of subthreshold depressions. Psychiatric Clinics of North America, 25(4), 685-698.

Kaviani, H., Gray, J. A., Checkley, S. A., Raven, P. W., Wilson, G. D., \& Kumari, V. (2004). Affective modulation of the startle response in depression: influence of the severity of depression, anhedonia, and anxiety. Journal of Affective Disorders, 83(1), 21-31.

Kemp, A. H., Felmingham, K., Das, P., Hughes, G., Peduto, A. S., Bryant, R. A., et al. (2007). Influence of comorbid depression on fear in posttraumatic stress disorder: an fMRI study. Psychiatry Research, 155(3), 265-269.

Kemp, A. H., Gray, M. A., Silberstein, R. B., Armstrong, S. M., \& Nathan, P. J. (2004). Augmentation of serotonin enhances pleasant and suppresses unpleasant cortical electrophysiological responses to visual emotional stimuli in humans. Neuroimage, 22(3), 1084-1096.

Kupfer, D. J., First, M. B., \& Regier, D. A. (2002). A research agenda for DSM- $V$. Washington: American Psychiatric Association.

Larson, C. L., Nitschke, J. B., \& Davidson, R. J. (2007). Common and distinct patterns of affective response in dimensions of anxiety and depression. Emotion, 7(1), 182-191.

Lovibond, P. F., \& Lovibond, S. H. (1995). The structure of negative emotional states: comparison of the Depression Anxiety Stress Scales (DASS) with the Beck Depression and Anxiety Inventories. Behaviour Research and Therapy, 33(3), 335-343.

Mathersul, D., Williams, L. M., Hopkinson, P. J., \& Kemp, A. H. 
(2008). Investigating models of affect: relationships among EEG alpha asymmetry, depression, and anxiety. Emotion, 8(4), 560-572.

Moffitt, T. E., Caspi, A., Harrington, H., Milne, B. J., Melchior, M., Goldberg, D., et al. (2007). Generalized anxiety disorder and depression: childhood risk factors in a birth cohort followed to age 32. Psychological Medicine, 37(3), 441-452.

Mogg, K., \& Bradley, B. P. (2005). Attentional bias in generalized anxiety disorder versus depressive disorder. Cognitive Therapy and Research, 29(1), 29 - 45.

Oke, A., Keller, R., Mefford, L., \& R., A. (1978). Lateralization of norepinephrine in human thalamus. Science, 200, $1411-1413$.

Papousek, I., \& Schulter, G. (2002). Covariations of EEG asymmetries and emotional states indicate that activity at frontopolar locations is particularly affected by state factors. Psychophysio$\log$, 39(3), 350-360.

Parker, G. (2000). Classifying depression: should paradigms lost be regained? American Journal of Psychiatry, 157, 1195-1203.

Phan, K. L., Wager, T., Taylor, S. F., \& Liberzon, I. (2002). Functional neuroanatomy of emotion: a meta-analysis of emotion activation studies in PET and fMRI. Neuroimage, 16(2), 331-348.

Reid, S. A., Duke, L. M., \& Allen, J. J. (1998). Resting frontal electroencephalographic asymmetry in depression: inconsistencies suggest the need to identify mediating factors. Psychophysiology, 35(4), 389-404.

Robinson, R. G. (1979). Differential behavioral amd biochemical effects of right and left hemispheric infarction in the rat. Science, 205, 707-710.

Sartorius, N., Ustun, T. B., Lecrubier, Y., \& Wittchen, H. U. (1996). Depression comorbid with anxiety: results from the WHO study on psychological disorders in primary health care. British Journal of Psychiatry Supplement(30), 38-43.

Shankman, S. A., \& Klein, D. N. (2003). The relation between depression and anxiety: an evaluation of the tripartite, approach- withdrawal and valence-arousal models. Clinical Psychology Review, 23, 605-637.

Surguladze, S., Brammer, M. J., Keedwell, P., Giampietro, V., Young, A. W., Travis, M. J., et al. (2005). A differential pattern of neural response toward sad versus happy facial expressions in major depressive disorder. Biological Psychiatry, 57(3), 201-209.

Wager, T. D., Phan, K. L., Liberzon, I., \& Taylor, S. F. (2003). Valence, gender, and lateralization of functional brain anatomy in emotion: a meta-analysis of findings from neuroimaging. $\mathrm{Neu}$ roimage., 19, 513-531.

Watson, D., Clark, L. A., Weber, K., Assenheimer, J. S., Strauss, M. E., \& McCormick, R. A. (1995). Testing a tripartite model: II. Exploring the symptom structure of anxiety and depression in student, adult, and patient samples. J Abnormal Psychology, 104(1), 15-25.

Watson, D., Weber, K., Assenheimer, J. S., Clark, L. A., Strauss, M. E., \& McCormick, R. A. (1995). Testing a tripartite model: I. Evaluating the convergent and discriminant validity of anxiety and depression symptom scales. Journal of Abnormal Psychology, 104(1), 3-14.

Williams, L. M. (2006). An integrative neuroscience model of "significance" processing. Journal of Integrative Neuroscience, 5(1), 1-47.

Williams, L. M., Kemp, A. H., Felmingham, K., Liddell, B. J., Palmer, D. M., \& Bryant, R. A. (2007). Neural biases to covert and overt signals of fear: dissociation by trait anxiety and depression. The Journal of Cognitive Neuroscience, 19, 1595-1608.

Williams, L. M., Phillips, M. L., Brammer, M. J., Skerrett, D., Lagopoulos, J., Rennie, C., et al. (2001). Arousal dissociates amygdala and hippocampal fear responses: evidence from simultaneous fMRI and skin conductance recording. Neuroimage, 14, 1070-1079.

Zald, D. H., Mattson, D. L., \& Pardo, J. V. (2002). Brain activity in ventromedial prefrontal cortex correlates with individual differences in negative affect. Proceedings of the National Academy of Sciences U S A, 99, 2450-2454. 